\title{
Direct photons and thermal dileptons: A theoretical review
}

\author{
Charles Gale ${ }^{a}$
}

${ }^{a}$ Department of Physics, McGill University, 3600 University St., Montreal, QC, H3A 2T8, Canada

We discuss the measurement of electromagnetic radiation produced in heavy ion collisions at SPS energies. We review the low invariant mass dilepton sector, the real photon data, and the spectra of intermediate mass dimuons. Along with this, we discuss the theoretical interpretations of those observables.

\section{Introduction}

The measurement of electromagnetic probes provides a valuable and a necessary complement to that of hadronic observables in an environment as potentially complex as that of ultrarelativistic heavy ion collisions. This fact owes partly to the weakness of final-state interactions in photon emission (real or virtual) but is also related to the richness of the information carried by the electromagnetic observables. Those statements can be made quantitative in the following. In a finite-temperature strongly-interacting medium the differential rate for real photon emission is given by [1,2]

$$
\omega \frac{d^{3} R}{d^{3} k}=-\frac{1}{(2 \pi)^{3}} \operatorname{Im} \Pi_{\mu}^{\mu} \frac{1}{e^{\beta \omega}-1},
$$

while that for virtual photon emission is

$$
E_{+} E_{-} \frac{d^{6} R}{d^{3} p_{+} d^{3} p_{-}}=\frac{2 e^{2}}{(2 \pi)^{6}} \frac{1}{k^{4}} L^{\mu \nu} \operatorname{Im} \Pi_{\mu \nu} \frac{1}{e^{\beta \omega}-1} .
$$

In the above equations $\omega$ is the photon energy, $\beta$ is the inverse temperature, $k^{2}$ is the virtual photon invariant mass, $L_{\mu \nu}$ is a lepton tensor, and the $E$ 's and $p$ 's are the lepton energy and momenta. $\Pi_{\mu \nu}$ is the retarded, finite-temperature photon self-energy: a direct window to the many-body physics. Vector Meson Dominance (VMD) couples the photon field (real or virtual) to hadronic matter in the confined sector via the current-field identity [3]: $J^{\mu}=\sum_{i} g_{i} \phi_{i}^{\mu}$, where the sum runs over the vector meson fields $\phi_{i}^{\mu}$. In the sector where the relevant degrees of freedom are partonic: $J^{\mu}=\sum_{q} e_{q} \bar{\psi}_{q} \gamma^{\mu} \psi_{q}$, where $e_{q}$ is a quark charge. In the region where confinement is effective, the dilepton production rate can then be directly related to the in-medium spectral density of the vector mesons. There lies one of the reasons for measuring and understanding the dilepton spectrum: the vector and axial vector correlators will mix via interactions with the thermal background [4]. In the limit of chiral symmetry restoration, those correlators are expected to become degenerate [5]. To obtain information on the axial current-current correlator through the 


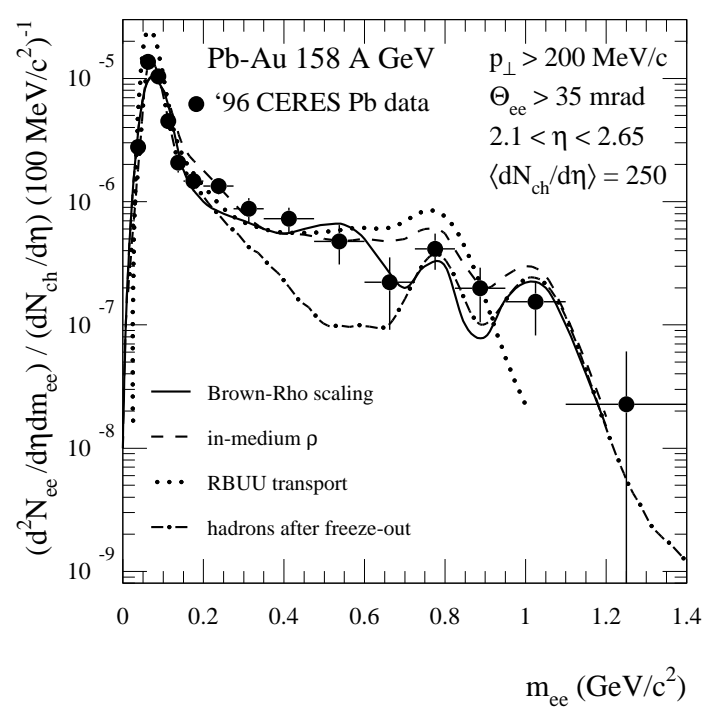

Figure 1. The low invariant mass CERES data with three theoretical curves representing models that are described in the text. The figure is from 13.

measurement of electromagnetic radiation constitutes a challenge to which we will return later. Also note that the importance of real photons and dileptons as tools for quark-gluon plasma (QGP) diagnostics was realized early on [6.7] and has fueled the interest in those variables ever since.

As RHIC begins its operation, it is sensible to assess the situation in terms of what has been measured and learnt so far at SPS energies. We shall discuss in turn the low dilepton invariant mass region, real photon measurements, and the intermediate dilepton invariant mass sector. It is futile here to attempt to cover and do justice to the many works that are relevant to this discussion. Also, in the spirit of a review with length constraints, we point to the appropriate references for the technically-intensive issues.

\section{Low mass dileptons}

This kinematical region, first explored in HELIOS dimuon measurements [8], was scrutinized by CERES in a series of dielectron measurements which have generated a considerable amount of theoretical activity [9,10]. The experimental measurements, together with some of their theoretical interpretations are shown in Fig. 1. As the status of the theoretical understanding of those inspiring data has not been profoundly modified since our last meeting in this series, there is no need here to go into a great deal of detail. The Brown-Rho scaling hypothesis is described in a recent review 11]. The curve labeled "in-medium $\rho$ " reflects an in-medium calculation of the vector meson spectral density [12], which is directly related to the dilepton emission rate through the current-field identity 
[2]. The results of a transport calculation involving an incoherent sum of meson reaction is also shown [14]. The "in-medium" calculation is a representation of fairly conventional many-body physics, as the vector mesons are broadened by interactions with nucleons and created mesons, as well as with the pions in the pion cloud. It turns out the the pole mass of the $\rho$ is not drastically modified by those interactions, owing to cancellations between channels 12,15]. QCD sum rules can only provide a broad consistency-check of some of the phenomenological models [16]. An experimental attempt to distinguish between the approaches listed above involves high resolution measurements at and around the vector meson peaks. The calculations relying on broadened spectral densities are lower there because of unitarity requirements [15,17]. Also, a study of the low mass dilepton excess as a function of the beam energy and of the baryon density would also constrain the models: runs at higher baryon densities may highlight novel many-body manifestations such as mixing effects [18]. Those important refinements of the data would help to bring this exciting chapter to some closure.

\section{Real photons}

The interest in the thermal emission of real photons partly stems from the seminal suggestion by Feinberg that thermal electromagnetic emission might be an important process when a large multiplicity of particles are produced in the final state [6]. To lowest order in the coupling constants, the fact that the photon spectrum carries with it information about the electromagnetic current-current correlator can be rewritten in the language of relativistic kinetic theory. In a quark-gluon plasma, the production of real photons will proceed through annihilation $(q \bar{q} \rightarrow \gamma g)$ and Compton $(g q \rightarrow \gamma q, g \bar{q} \rightarrow \gamma \bar{q})$ channels. Therefore, the photons produced in the plasma carry information on the thermodynamic state of the parton medium at the moment of their production. However from a practical point of view, the signal-to-background ratio is smaller than that for dileptons by roughly two orders of magnitude, due especially to the large $\pi^{0}$ and $\eta$ decay contributions. This fact makes the real photon measurements particularly challenging. At SPS energies, the first attempts to observe direct photon production in ultrarelativistic nuclear collisions with $\mathrm{O}$ and $\mathrm{S}$ beams found no significant excess [19]. The WA80 collaboration has obtained upper bounds for the $p_{T}$ spectrum of real photons generated in $\mathrm{S}+\mathrm{Au}$ collisions

at $200 \mathrm{AGeV}$ [20]. A measurement of direct photons in ${ }^{208} \mathrm{~Pb}+{ }^{208} \mathrm{~Pb}$ collisions at 158 $\mathrm{AGeV}$ was finally reported by WA98 [21].

Recent times have witnessed a flurry of theoretical activity centered on the theoretical interpretation of the WA98 photon measurements. The data along with a pQCD estimate are shown in Fig. 2. It is impossible to discuss here the many calculations, but ingredients of some theoretical interpretations are not free from ambiguities and those should be pointed out in order to make progress. Let us first consider the high transverse momentum part of the measured photon spectrum. In hadron-hadron collisions at ISR energies, comparisons of the measured high $p_{T}$ sector with NLO-QCD direct photon calculations reveal that pQCD contributions are quantitatively important [23]. This component of the spectrum should reflect the parton distribution functions. However, despite many years of experimental and theoretical effort, the process of inclusive photon production in the collision of elementary hadrons is not yet fully understood, and those uncertainties 


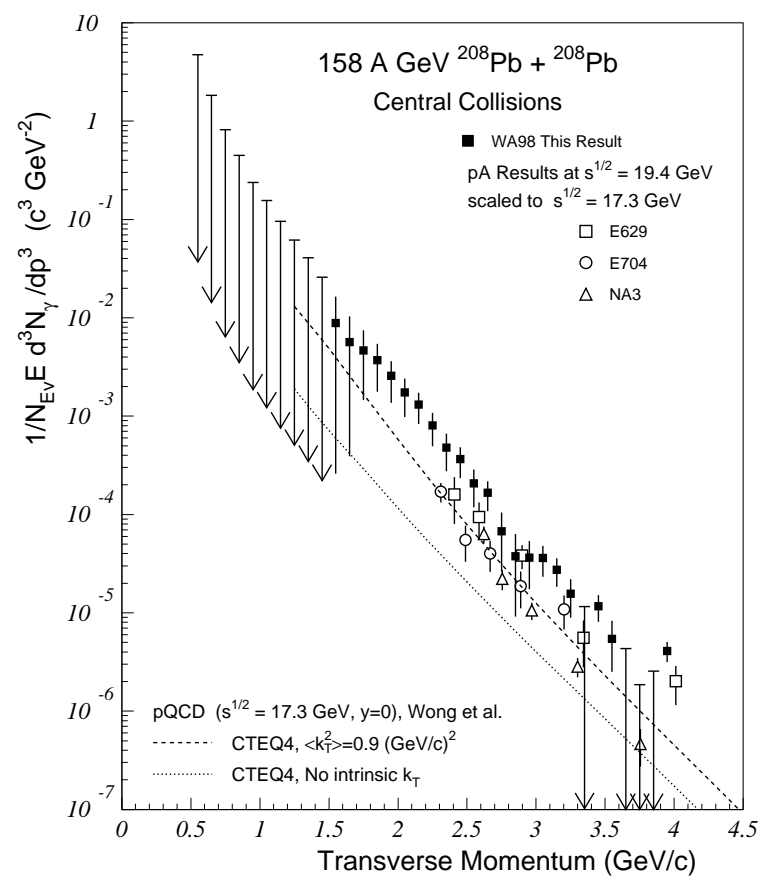

Figure 2. The WA98 real photon invariant cross section data, as a function of photon transverse momentum [21]. The pQCD calculation with intrinsic $k_{\perp}$ is from [22].

propagate to nuclear collisions. A recent critical survey concludes that the present fixed target data on inclusive prompt photon data at ISR energies $(\sqrt{s} \geq 23 \mathrm{GeV})$ are inconsistent [24], making it difficult to extract from them physically meaningful values of model parameters. Bearing those uncertainties in mind, the idea of attributing the pattern of deviations between the measured direct photon cross sections and NLO calculations to parton transverse momentum (neglected in the NLO calculations) has however gathered support 25,26]. Taking a confinement radius of $0.5 \mathrm{fm}$, the intrinsic transverse momentum generated thusly is $\left\langle k_{\perp}^{2}\right\rangle \simeq 0.32(\mathrm{GeV} / \mathrm{c})^{2}$. Furthermore, soft-gluon emission will make this value larger [27]. The resummation calculation for multiple soft-gluon emission in direct photon production is challenging, and part of their contribution will receive a non-perturbative component at the $\sqrt{s}$ values that are relevant for this discussion. As a temporary substitute for a rigorous calculation, effects of soft-gluon radiation are approximated by a convolution of the LO cross section with a $k_{\perp}$-smearing function. Early estimates obtained $<k_{\perp}^{2}>\simeq 0.9(\mathrm{GeV} / \mathrm{c})^{2}$ at $\sqrt{s}=20 \mathrm{GeV}$ 28.

In addition to these considerations (and to their inherent uncertainties), multiple initial state scattering (the Cronin effect) in pA and AA collisions will add to the parton transverse momenta [29, 30], prior to the photon-forming interaction. Clearly, before a consensus is achieved, systematic studies of hadron and photon spectra are needed in pp, pA, and AA collisions at the same energies. This has not been possible in the past, and the necessary energy-scaling procedures add to the ambiguities. This situation will not be repeated at RHIC, where all three types of collisions will be accessible to a given 
experimental setup. One may then ask how crucial are the high temperatures advocated by early hydrodynamical calculations [31]? As long as the quantitative role played by parton momentum broadening in nuclear collisions is not fully elucidated, it appears difficult to bring this issue to closure. The global survey suggested above would go a long way in this direction. A closely related issue is the use in the deconfined-QCD sector of the photon production rates obtained from two-loop topologies at finite-temperature 31, 32. Those utilize the hard thermal loop resummation method in finite temperature perturbation theory [33]. This technique allows the investigation of screening effects in a systematic way. At the one-loop level, this approach was successfully used to compute the emission rate of hard real photons [34,35] and dileptons [36]. The two-loop rates receive a large boost from a colinear singularity, making the process labeled "annihilation-withscattering" dominant [32]. Some progress has been made in understanding those results in terms of an ordering of the different length scales in the problem [37]. However, even including obvious coupling constants scaling, it is fair to say that the use at the SPS of the photon rates calculated for asympotically high energies rests on the belief that the relative contributions to the photon spectrum of the different self-energy cuts are generic. This still remains to be put on a firm theoretical foundation.

The low $p_{T}$ part of the direct photon spectrum is linked to softer physical contributions. Those typically will involve interactions between degrees of freedom from the lower temperature confined sector. Let's consider the kinetic-theory representation of the hadronic reactions responsible for direct photon production. Those rates have been evaluated first in Ref. [35], have been verified subsequently [38, 39], and involve a gas of $\pi, \eta, \rho$, and $\omega$ mesons. Also, contributions involving the $a_{1}$ have been shown to be quantitatively important [40]. Herein lies an ambiguity in the hadronic phase: there is no unique way to implement a chirally-symmetric model with vector mesons. A consequence of this is that a survey of the literature rapidly reveals that photon production rates in a meson gas can differ by factors of $\sim 3$, at temperatures and transverse momenta that are germane to the conditions discussed here 40,41. We advocate again a systematic treatment of all the available hadronic data in order to reduce the degeneracies in parameter-space of the effective theories involved [42]. Furthermore, definite conclusions about the nature of the sources in the soft part of the photon spectrum produced in heavy ion collisions are hindered by the following fact. Consider for a moment the WA80 photon data $(\mathrm{S}+\mathrm{Au}$, at $200 \mathrm{AGeV}$ ), together with photon rates calculated with the same vector meson spectral density as that used to interpret the CERES data. The photon spectrum one obtains after a simple fireball space-time evolution constitutes at least $\sim 16 \%$ of the signal at $p_{T}$ $=1.5 \mathrm{GeV} / \mathrm{c}$ [12. It is known that the in-medium effects in this energy regime are largely baryon-driven [12]. This begs the question of how much is the corresponding contribution to the WA98 $\mathrm{Pb}+\mathrm{Pb}$ data, as the appropriate calculation has not yet been done. There, an examination of the data with a LO-pQCD calculation with an intrinsic $\left\langle k_{\perp}^{2}>\approx 1\right.$ $(\mathrm{GeV} / \mathrm{c})^{2}$ yields $\sim 45 \%$ of the measured yield at $p_{T}=2.5 \mathrm{GeV} / \mathrm{c}$ [22]. Barring the Cronin effect, the sources needing to be added then are the contribution from the in-medium vector meson spectral densities and the additional contributions from the meson gas that are not included in them as, in self-energy parlance, they are specifically two-loop contributions. This course of action is then laid out and preliminary results are promising [43]. 


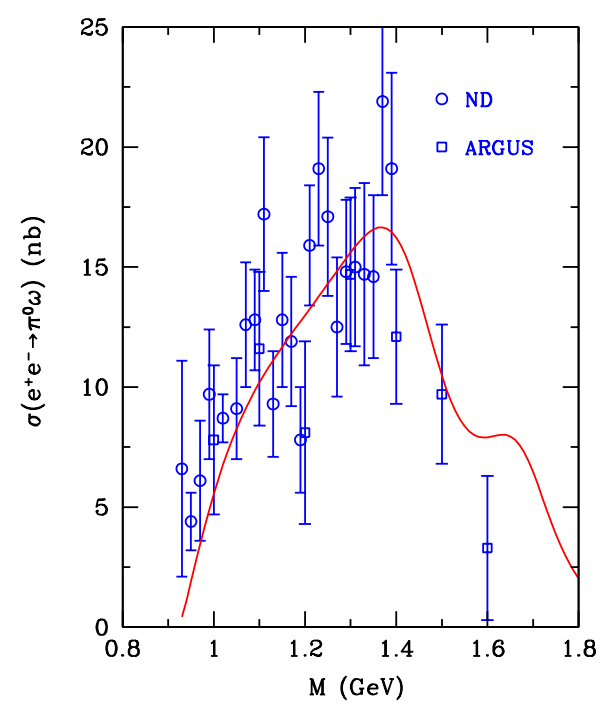

Figure 3. The cross section for $e^{+} e^{-} \rightarrow \pi^{0} \omega$ as a function of dilepton invariant mass. The solid curve shown here is based on the model of [47]. The experimental data are from the ND [47] and ARGUS [50] collaborations.

Is therefore appears that uncertainties remain in the theoretical interpretation of the direct photon spectrum, both in the low and high transverse momentum regions. However, several of those difficulties can be resolved.

\section{Intermediate mass dileptons}

Dimuon pairs in the intermediate invariant mass region $\left(m_{\phi}<M<m_{J / \psi}\right)$ have been measured at CERN by the Helios-3 [44] and NA38/NA50 collaborations [45]. We consider those in turn. The Helios-3 data is concerned with dimuon production in $\mathrm{p}+\mathrm{W}$ and $\mathrm{S}+$ W collisions at $200 \mathrm{AGeV}$. As in the low mass sector, an analysis of the heavy ion data and of the proton-nucleus data revealed an excess of lepton pairs over that expected from known sources; those being the direct decays of primary vector mesons, the Drell-Yan contribution and the correlated semileptonic decay of open charm mesons. Naturally, one should ask whether there exist mesonic channels similar to the ones at play in the low invariant mass region that could significantly contribute in this kinematical region. However a precise determination of the specific channels starting from effective chiral Lagrangian techniques remained elusive, as in this invariant region the basic VMD form factors will be significantly off-shell and there is enough phase space for the initial channels to couple to a variety of high mass vector mesons [46]. Fortunately, there exists a wealth of data concerning $e^{+} e^{-} \rightarrow$ hadrons in the appropriate invariant mass range [47. Those data can be analyzed channel-by-channel [48]: an example appears in Fig. 3. Inverting the channels that have two-body mesonic final states, a reaction database can be constructed. Those contributions can then be used in a transport approach that can be directly compared 


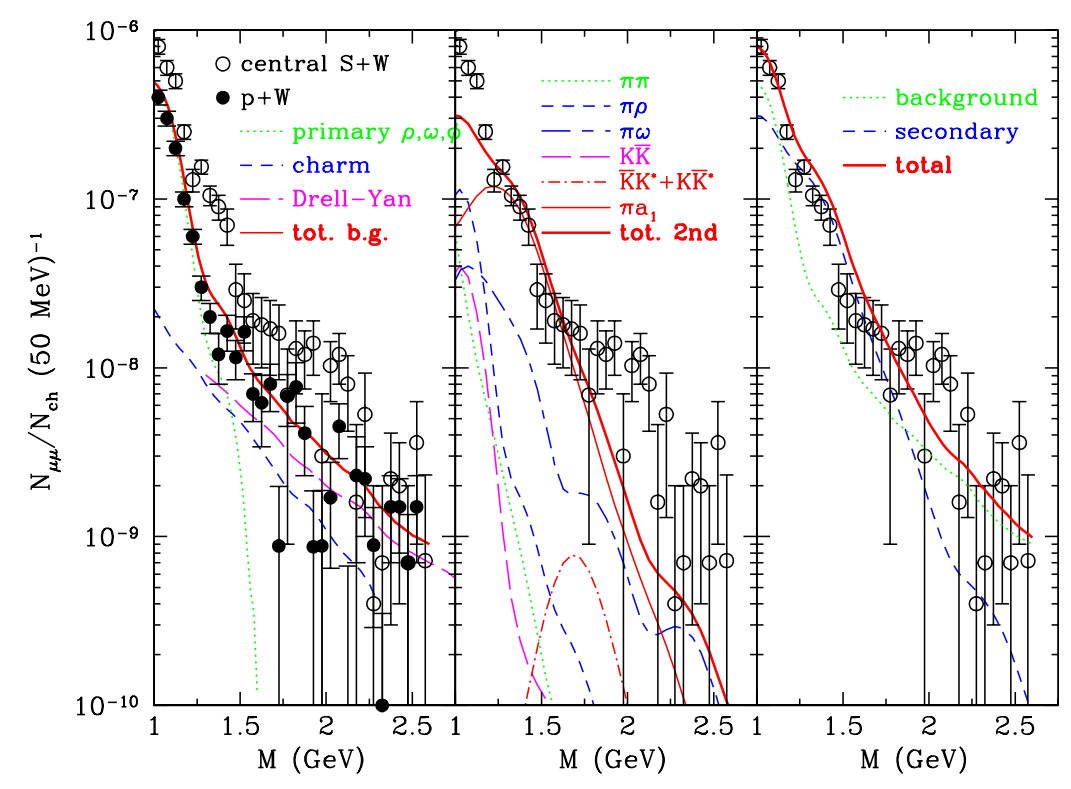

Figure 4. Left panel: comparison of background, Drell-Yan, and open charm decays with $\mathrm{p}+\mathrm{W}$ and $\mathrm{S}+\mathrm{W}$ Helios-3 dimuon data. Middle panel: some mesonic reactions contributing to lepton pair final states are shown. Right panel: the sum, the background, and the secondary contributions are shown with the data from central $\mathrm{S}+\mathrm{W}$ collisions.
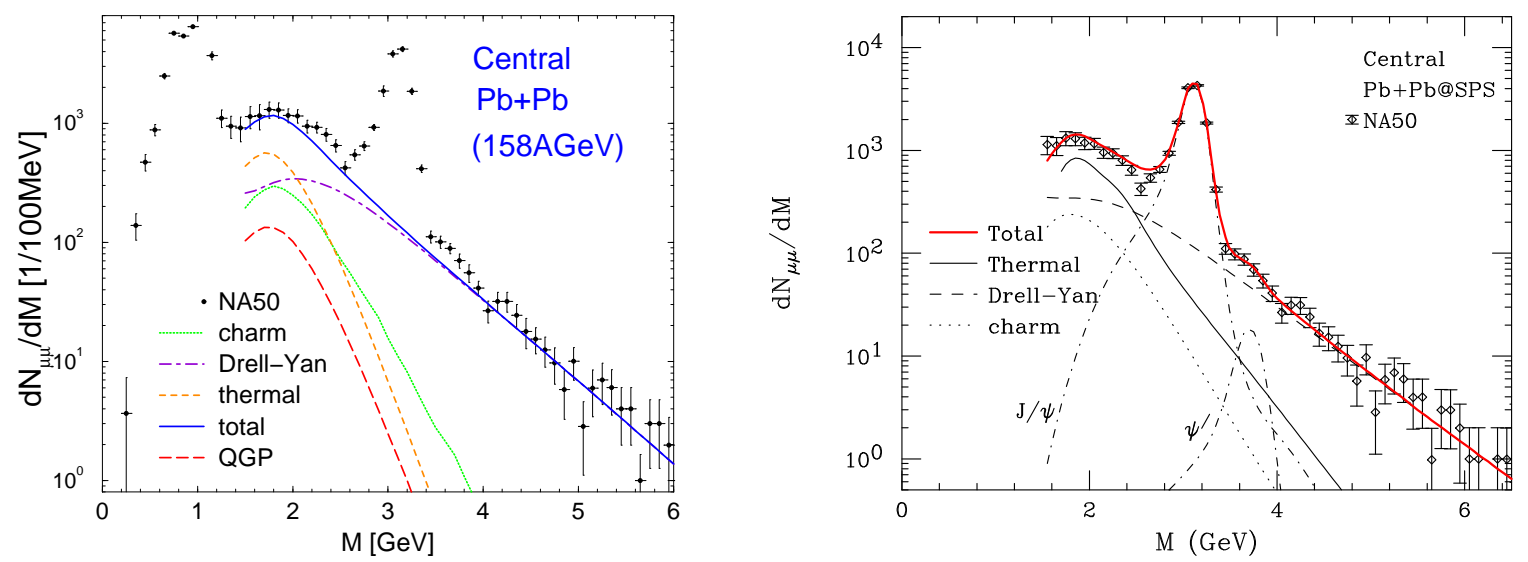

Figure 5. Comparison with the NA50 intermediate mass dimuon data. The left panel is a calculation from Ref. [51]. The right panel is a calculation from Ref. [52]. There, the "thermal" contribution includes the QGP component.

with experimental measurements. The procedure described here was used in conjunction with a cascade-type model and compared with the Helios-3 data [49], and some results are shown in Fig. 由. It appears that once the "thermal" channels are included, the measured observable calls for little or no additional sources. Recently, this approach was applied to 
the intermediate mass dimuon data measured by NA50 [51]. A result of this calculation is shown in Fig. 5, together with the result of a similar approach. Those two calculations differ mainly in the dynamical models used to integrate out the dilepton rates, and in their quark-gluon plasma content. Whereas a study is underway [53 to try and pin down, as much as the data allows, model parameters such as the initial temperature (while still being consistent with the global set of measured observables at those bombarding energies), an important message emerges: the importance of the secondary mesonic reactions [49] can not be denied. To completely rule out a mechanism like $c \bar{c}$ enhancement, for example, a better measurement is needed and a dedicated experiment is in the planning stages 54 .

\section{Conclusion}

The measurement of electromagnetic radiation at the SPS has generated exciting data that has considerably motivated and stirred the field as a whole. It is appropriate to pause at this point and to reflect on what has been learnt. The low mass dileptons show strong indications of the signature of a genuine, in-medium many-body effect. This is an achievement that should not be underplayed. As elaborated on in the text, the theoretical effort on this is ongoing and high resolution data at the vector meson masses will bring this discussion to a new level of quantitative assessment. In the real photon sector, a systematic evaluation of the nuclear $k_{\perp}$ broadening in hadron and electromagnetic spectra is called for. Similarly, the theoretical framework used for real photons has to be consistent, and in fact identical, with that used in the virtual photon domain. This is vital for the sake of theoretical consistency and also in view of the fact that those data do show common global features [55]. Some issues discussed here represent well-posed problems and answers are to be expected soon.

Have we seen a restoration (even partial) of chiral symmetry? Without a window on the axial correlator this issue remains inconclusive. This will be difficult to assess from studies of low mass dileptons as the axial vector channel is far below those of stronger sources [14, [15]. However, we suggest that it might be possible in real photon and/or intermediate mass dilepton measurements, as there the contribution of the $a_{1}$, for example, is not small [40,49]. Before this can be done, the calculations of the axial vector spectral density have to be brought to a level of sophistication close to those that exist in the vector channel. Have we seen a quark-gluon plasma? As it is, some analyses of the hard portion of the photon spectrum and of the intermediate mass dilepton data do contain a QGP component, albeit at different levels. In my opinion is it still too early to tell, but keeping in mind the caveats outlined in this talk, it is fair to say that this carries the suggestion that the SPS has ventured in a threshold energy density region. RHIC has the tantalizing mandate to go deeper in this new realm.

\section{Acknowledgments}

It is a pleasure for me to thank my collaborators. This work is supported in part by the Natural Sciences and Engineering Research Council of Canada, and in part by the Fonds FCAR of the Quebec Government. 


\section{REFERENCES}

1. L. D. McLerran and T. Toimela, Phys. Rev. D 31 (1985) 1545; H. A. Weldon, Phys. Rev. D 42 (1990) 2384.

2. Charles Gale and Joseph I. Kapusta, Nucl. Phys. B357 (1991) 65

3. J. J. Sakurai, Currents and Mesons, (University of Chicago Press, Chicago, 1969); H. B. O'Connell, B. C. Pearse, A. W. Thomas, and A. G. Williams, Prog. Part. Nucl. Phys. 39 (1997) 201.

4. M. Dey, V. Eletsky and B. I. Ioffe, Phys. Lett. B252 (1990) 620.

5. J. I. Kapusta and E. V. Shuryak, Phys. Rev. D 49 (1994) 4694.

6. E. L. Feinberg, Nuovo Cimento 34 A (1976) 391.

7. E. V. Shuryak, Phys. Lett. B78 (1978) 150.

8. A. L. S. Angelis, Eur. Phys. J. C 13 (2000) 433, and references therein.

9. B. Lenkeit for the CERES collaboration, Nucl. Phys. A661 (1999) 23c, and references therein.

10. H. Appelshaeuser, these proceedings.

11. G. E. Brown and Mannque Rho, hep-ph/0103102, and references therein.

12. R. Rapp and J. Wambach, Adv. Nuc. Phys. 25 (2000) 1 [hep-ph/9909229].

13. I. Tserruya, nucl-ex/9912003.

14. V. Koch and C. Song, Phys. Rev. C 36 (1996) 1903.

15. Ralf Rapp and Charles Gale, Phys. Rev. C 60 (1999) 024903.

16. S. Leupold, W. Peters, and U. Mosel, Nucl. Phys. A628 (1998) 311.

17. W. Cassing and E. L. Bratkovskaya, Phys. Rep. 308 (1999) 65.

18. G. Wolf, B. Friman, and M. Soyeur, Nucl. Phys. A640 (1998) 129; O. Teodorescu, A. K. Dutt-Mazumder, and C. Gale, Phys. Rev. C 61 (2000) 051901; Phys. Rev. C 63 (2001) 034903.

19. T. Åkesson et al., Z. Phys. C 46 (1990) 369; R. Albrecht et al., Z. Phys C 51 (1991) 1; R. Baur et al., Z. Phys. C 71 (1996) 571.

20. R. Albrecht et al., Phys. Rev. Lett. 76 (1996) 3506.

21. M. M. Agarwal et al., Phys. Rev. Lett. 85 (2000) 3595; nucl-ex/0006007; A. Lebedev, these proceedings.

22. C. Y. Wong and H. Wang, Phys. Rev. C 58 (1998) 376.

23. P. Aurenche, R. Baier, M. Fontannaz, and D. Schiff, Nucl. Phys. B286 (1987) 509; Nucl. Phys. B297 (1988) 661.

24. P. Aurenche et al., Eur. Phys. J. C 9 (1999) 107.

25. J. Huston et al., Phys. Rev. D 51 (1995) 6139.

26. L. Apanasevich et al., Phys. Rev. D 59 (1999) 074007.

27. J. C. Collins, D. E. Soper, and G. Sterman, Nucl. Phys. B250 (1985) 199.

28. J. Owens, Rev. Mod. Phys. 59 (1987) 465.

29. X. N. Wang, Phys. Rev. C 61 (2000) 064910.

30. G. Papp, P. Levai, and G. Fai, Phys. Rev. C 61 (2000) 021902; G. Papp et al., these proceedings.

31. Dinesh Kumar Srivastava and Bikash Sinha, nucl-th/0006018.

32. P. Aurenche, F. Gélis, R. Kobes, H. Zaraket, Phys. Rev. D 58 (1998) 085003.

33. R. D. Pisarski, Nucl. Phys. B309 (1988) 476; Phys. Rev. Lett. 63 (1989) 1129; Nucl. 
Phys. A525 (1991) 175c; E. Braaten and R. Pisarski, Phys. Rev. Lett. 64 (1990) 1338; Nucl. Phys. B337 (1990) 569; Nucl. Phys. B339 (1990) 310; J. Frenkel and J. C. Taylor, Nucl. Phys. B334 (1990) 199.

34. R. Baier, H. Nakkagawa, A. Niégawa, and K. Redlich, Z. Phys. C 53 (1992) 433.

35. Joseph Kapusta, Peter Lichard, and David Seibert, Phys. Rev. D 44 (1991) 2774.

36. E. Braaten, R. Pisarski, and T. C. Yuan, Phys. Rev. Lett. 64 (1990) 2242; S. M. H. Wong, Z. Phys. C 53 (1992) 465; T. Altherr and P. V. Ruuskanen, Nucl. Phys. B380 (1992) 377; Markus H. Thoma and Christoph T. Traxler, Phys. Rev. D 56 (1997) 198. 37. F. Gélis, these proceedings.

38. S. Sarkar, J. Alam, P. Roy, A. K. Dutt-Mazumder, B. Dutta-Roy, and B. Sinha, Nucl. Phys. A634 (1998) 206.

39. M. Bertrand, MSc Thesis (McGill University, 2001), unpublished.

40. L. Xiong, E. Shuryak, and G. Brown, Phys. Rev. D 46 (1992) 3798; C. Song, Phys. Rev. C 47 (1993) 2861; C. Song, C. M. Ko, and C. Gale, Phys. Rev. D 50 (1994) 1827.

41. J. Steele, H. Yamagishi, and I. Zahed, Phys. Lett. B384 (1996) 255; Miklos A. Halasz, James V. Steele, Guo-qiang Li, Gerald E. Brown, Phys. Rev. C 58 (1998) 365.

42. See, for example, Song Gao and Charles Gale, Phys. Rev. C 57 (1998) 254.

43. C. Gale, M. A. Halasz, and R. Rapp, in preparation.

44. M. Masera, Nucl. Phys. A590 (1995) 93c.

45. P. Bordalo, Nucl. Phys. A661 (1999) 538c.

46. Particle Data Group, Eur. Phys. J. C 15 (2000) 1.

47. S. I. Dolinsky et al., Phys. Rep. 202 (1991) 99.

48. I. Kvasnikova, PhD Thesis (McGill University, 2001), unpublished.

49. G. Q. Li and C. Gale, Phys. Rev. Lett. 81 (1998) 1572; Phys. Rev. C 58 (1998) 2914.

50. N. Albrecht et al., Phys. Lett. B185 (1987) 223.

51. Ralf Rapp and Edward Shuryak, Phys. Lett. B473 (2000) 13.

52. D. K. Srivastava et al., these proceedings.

53. I. Kvasnikova, C. Gale, D. K. Srivastava, and B. Sinha, in preparation.

54. C. Lourenço, these proceedings.

55. B. Kampfer et al., these proceedings. 